\title{
Modelling Motor Vehicle Emissions and Population Exposure in South Australia
}

\author{
$\underline{\text { L. Schultz }}^{\text {a }}$, B. Chiera ${ }^{\mathrm{a}}$, P. Shah ${ }^{\mathrm{b}}$, J. Boland ${ }^{\mathrm{a}}$ \\ ${ }^{a}$ School of Information Technology \& Mathematical Sciences, University of South Australia \\ ${ }^{\mathrm{b}}$ Air \& Noise Group, Science \& Assessment Division, South Australian Environment Protection Authority \\ Email: Lisa.Schultz@mymail.unisa.edu.au
}

\begin{abstract}
Motor vehicle exhaust has been identified as a dominant source of anthropogenic ambient atmospheric pollution. The greatest concentrations of traffic-related air pollution (TRAP) are emitted along heavily trafficked major roadways from which the associated detrimental effects of TRAP exposure have been wellestablished (HEI Panel on the Health Effects of Traffic-Related Air Pollution (2010)). Consequently, populations residing near major roadways have the greatest likelihood of potential exposure to TRAP and a high risk of experiencing adverse health effects.
\end{abstract}

We investigate pollution exposure levels for Adelaide residents in heavily trafficked areas by modelling and simulating TRAP emissions and movement in the atmosphere, with the development of an enhanced Link Emissions Modelling procedure for South Australia. The current Link Emissions Model (CLEM) for South Australia uses emission factors developed overseas and is based on a single average speed. The enhanced Link Emissions Model uses petrol passenger vehicle emissions and vehicle speed data from the second National InService Emissions study, to derive new Australian emission factors for use in South Australia. The result is the petrol passenger vehicle Link Emissions Model, PLEM. In essence, PLEM is an augmented version of CLEM, however provides the advantages of accommodating a range of vehicle speeds and is based on Australian data. The enhanced Link Emissions Modelling procedure for South Australia estimates road link TRAP emissions through the use of PLEM for petrol passenger vehicles in conjunction with CLEM for all other vehicle types.

The enhanced Link Emissions Model is implemented in a case study of selected densely trafficked roadways near residential areas of Adelaide, South Australia, to assess population TRAP exposure levels and the current air quality status in Adelaide. Vehicular exhaust emissions are estimated for each road link in the case study area using the enhanced Link Emissions Model. The Air Pollution Model (TAPM), an air quality tool for pollution dispersion, is used to analyse the movement of the link emission estimates in the atmosphere with pollution exposure maps showing approximate pollution concentrations for the case study region, generated by combining TAPM output with Geographic Information Systems data. Results of the case study indicate that TRAP concentrations are greatest over main roads and their intersections, with pollutant concentrations declining with increasing distance from the main roads. Pollution concentrations fluctuate across the week, with each day typically experiencing two peaks in air pollution concentrations corresponding to peak traffic hours. The lowest pollution concentrations occur during the early hours of a day when traffic volumes are typically lowest. Furthermore, the results suggest TRAP concentrations are subject to seasonal variation with the greatest concentrations occurring during the calm, cold weather conditions of winter and the lowest concentrations occurring during the windy, warm summer season. Atmospheric vehicle exhaust pollution concentrations in the case study area of Adelaide are comfortably below the threshold levels specified by the World Health Organisation however Adelaide residents are exposed to a notable volume of vehicular exhaust pollution. Thus mitigation strategies for TRAP emission and exposure should be designed and implemented for South Australia.

The case study results indicate use of the enhanced Link Emissions Model provides a strong understanding of approximate pollution concentrations in the Adelaide air shed, forming a solid platform upon which to base future informed strategies for emission reduction and exposure mitigation.

Keywords: Motor vehicles, emissions, pollution, dispersion, exposure 


\section{INTRODUCTION}

Motor vehicle exhaust is a major contributor to global ambient anthropogenic urban air pollution (Fenger, 1999) with the detrimental health effects resulting from exposure to this pollution now well-established (HEI Panel on the Health Effects of Traffic-Related Air Pollution, 2010). Exposure to traffic-related air pollution (TRAP) has been associated with a large number of health issues including an increased risk of cardiopulmonary and cardiovascular disease (Miller et al., 2007), decreased mental function in older adults (Power et al., 2011), increased occurrence of asthmatic episodes and respiratory infection among children (Brauer et al., 2002), adverse birth outcomes (Gehring et al., 2011), prevalence of type 2 diabetes (Raaschou-Nielsen et al., 2013) and premature mortality (Scoggins et al., 2004). Moreover, proximity to the pollution source affects the severity of the health effect (Oosterlee et al., 1996) and as such, populations residing near densely trafficked roads are at greater risk of experiencing poor health episodes associated with TRAP exposure.

An accurate understanding of the quantity and movement of atmospheric pollution is essential in order to successfully design and implement appropriate air quality control strategies and mitigate TRAP exposure. Governments commission the development of Link Emissions Models which are used to estimate road link TRAP emissions by combining traffic activity data, such as vehicle fleet size and composition, with emission factors, which describe exhaust pollution emission rates. Emission factors are typically defined for categories of vehicles based on vehicle characteristics including age and engine size. The current Link Emissions Model (CLEM) used by the South Australian Environment Protection Authority employs overseas emission factors that are calibrated by a constant for adapted use in South Australia. Moreover, CLEM assumes all traffic flows at a constant speed, which may not necessarily be an accurate assumption in emissions modelling as pollutant emission rates vary with vehicular speed (Van Mierlo et al., 2004).

We investigate South Australian TRAP concentrations and exposure levels in residential areas near major, densely trafficked roads, by developing an enhanced Link Emissions Model for South Australia. This approach employs Australian petrol passenger vehicle emissions data from the second National In-Service Emissions (NISE2) study to develop a new Link Emissions Model called PLEM, designed specifically for petrol passenger vehicles. In practice, PLEM is used in conjunction with CLEM to estimate TRAP emissions from all vehicle types. The benefits of the enhanced model include the usage of recently collected Australian data and the incorporation of a range of vehicle speeds, to produce a more realistic representation of driving behaviours.

This paper is outlined as follows. In Section 2, details of the NISE2 petrol passenger vehicle data used to develop PLEM are given and in Section 3 we describe the enhanced Link Emissions Model. In Section 4 the enhanced Link Emissions Model is implemented for a selection of major roads prone to large traffic volumes in the vicinity of residential areas of Adelaide, South Australia. We then assess current population TRAP exposure levels in these areas. Finally, in Section 5 conclusions are drawn on the current air quality status and population pollutant exposure levels for residential areas near main roads in Adelaide.

\section{THE SECOND NATIONAL IN-SERVICE EMISSIONS DATA}

To date, emission factors used in South Australian Link Emissions Modelling were based on a single mean speed and developed using overseas traffic conditions. The petrol passenger vehicle emissions data from the second National In-Service Emissions (NISE2) Australian study has provided an opportunity to derive Australian-based petrol passenger vehicle emission factors and subsequently develop a petrol passenger vehicle Link Emissions Model (PLEM). In this section we provide further details on the NISE2 data.

The NISE2 study (Department of the Environment, Water, Heritage and the Arts, 2009) is a major study periodically conducted by the Australian government to assess on-road vehicle compliance with the emissions standards specified by the Australian Design Rules (ADR). The NISE2 study measured vehicle emissions of carbon monoxide (CO), total hydrocarbons (THC) and oxides of nitrogen (NOx) from a fleet of 503 petrol passenger vehicles. The study inherently classified each participating vehicle according to engine size as either large, medium or small passenger vehicles (PVL, PVM, or PVS, respectively), large or compact sports utility vehicles (SUV-L or SUV-C, respectively) or light commercial vehicles (LCV).

We further classify the NISE2 vehicles into four age groups based on ADR emissions legislative periods, yielding 24 vehicle categories. We will model the emissions for each of the three pollutants over the 24 vehicle categories, thereby producing 72 new Australian emission factors. As we do not have emissions data for any other vehicle types, we are currently only able to derive new petrol passenger vehicle emission factors. In the future, as data becomes available we will develop new emission factors for other vehicle types. 
The NISE2 emissions data was available at non-integer speed values, however to avoid sensitivity to noise in the emission factors we will derive, the emissions data was aggregated at $2 \mathrm{~km} / \mathrm{hr}$ speed intervals. The aggregated NISE2 emissions data for each vehicle in a given vehicle category was then combined using a proportionally weighted average model to obtain a single emissions data set.

The NISE2 study also contained a Composite Urban Emissions Drive Cycle (CUEDC) which measured Australian vehicle speed data for four different road types to obtain vehicle speed profiles typical of arterial, congested and residential roads as well as freeways. In previous work (Schultz et al., 2011), we modelled the vehicle speed data for each of these road types to obtain vehicle speed distributions which will be used by PLEM. As the emission factors of CLEM are based on a single mean speed for all road types, it was not necessary to incorporate these vehicle speed distributions into this model.

\section{ENHANCED VEHICLE EMISSIONS MODELLING PROCEDURE}

The enhanced Link Emissions Modelling procedure for South Australia is summarised in Figure 1. The original CLEM model is shown (solid lines (-)) with PLEM providing the augmentation (dashed (- -) components). There are two main phases in the enhanced Link Emissions Modelling procedure: the modelling phase, in which road link TRAP emissions are estimated; and the scenario analysis phase which analyses atmospheric dispersion of the link emission estimates under different meteorological scenarios.

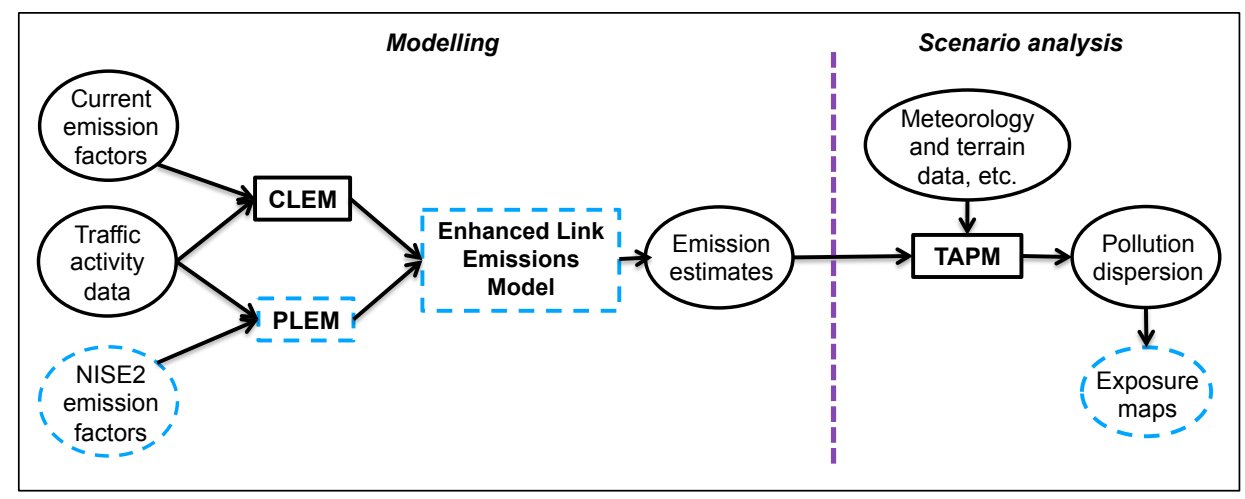

Figure 1. Methodology framework indicating CLEM (solid - black lines) and PLEM (dashed - - blue lines).

The first stage of the modelling phase (Figure 1) was the derivation of NISE2 emission factors for petrol passenger vehicles (to be detailed in Section 3.1). We then developed a South Australian petrol passenger vehicle Link Emissions Model (PLEM) which we will outline in Section 3.2. The enhanced Link Emissions Model uses PLEM in conjunction with CLEM, to estimate TRAP emissions from all vehicular sources.

The scenario analysis phase (Figure 1) provides a complete analysis of atmospheric vehicle exhaust pollution. The Air Pollution Model (TAPM) is employed to analyse the movement of the link emission estimates in the atmosphere by using terrain and historical meteorological data for a specified time period (Hurley, 2008). Combining TAPM output with Geographic Information Systems (GIS) data yields pollutant exposure maps used to identify areas of high TRAP concentrations and assess population TRAP exposure levels.

\subsection{Emission Factor Modelling}

To derive new petrol passenger vehicle emission factors we employed Local Polynomial Regression to model pollutant emissions for each vehicle category of the NISE2 data. Local Polynomial Regression was an attractive choice as it uses the local structure of the data to construct the regression estimate (Loader, 1999). As such, Local Polynomial Regression is an ideal modelling choice for complex data structures which do not necessarily conform to the predefined functions of traditional regression models. Moreover, the only user-input required for Local Polynomial Regression is the specification of the nearest neighbour fraction, $\alpha \in[0,1]$, which governs the degree of smoothing in the Local Polynomial Regression model. 
In producing a Local Polynomial Regression model, a weighted polynomial, $Y_{k}$, is fitted to data points in a neighbourhood around the fitting point $x_{k}, k=1, \ldots, n$, such that

$$
Y_{k}=\mu\left(x_{k}\right)+\epsilon_{k}
$$

where $\mu\left(x_{k}\right)$ is an unknown function which we assume can be approximated by a polynomial and $\epsilon_{k}$ are the residual errors, assumed to be independent and identically distributed with zero mean and finite variance.

The width of the local neighbourhood around the fitting point $x_{k}$, known as the nearest neighbour bandwidth $h\left(x_{k}\right)$, is based on the nearest neighbour fraction $\alpha \in[0,1]$ which stipulates the proportion of the sample data to be contained within the local neighbourhood. As such, $h\left(x_{k}\right)$ varies for each fitting point $x_{k}$. When data points are sparsely distributed, the local neighbourhood is large, yielding a wider, flatter estimate of $\mu(x)$. Conversely, densely distributed data results in a larger, narrower estimate of $\mu(x)$. Data points that lie within $h\left(x_{k}\right)$ are weighted such that observations near the fitting point have a stronger influence on the shape of $\mu\left(x_{k}\right)$ than data points further away. We used the traditional Tricube weight function (Loader, 1999)

$$
w_{k}(x)=\left(1-\left|\frac{x_{k}-x}{h\left(x_{k}\right)}\right|\right)^{3} .
$$

Finally, we approximate $\mu\left(x_{k}\right)$ by a quadratic polynomial using the least squares method (Loader, 1999).

Due to space constraints we have not included the results of the emission factor modelling process here. However, each of the 72 new emission factors derived passed a number of statistical tests and model validation diagnostics without serious issue. In general, CO emissions proved the most challenging to model over all 24 vehicle categories, particularly for older vehicles, often requiring a square-root or logarithmic transformation of the data. Emissions of NOx and THC were more agreeable to the modelling process, with THC seldom requiring any transformation.

\subsection{Link Emissions Model for South Australia}

The second part of the modelling phase (Figure 1) focused on developing road link TRAP emissions. The enhanced Link Emissions Model has two main components:

1. CLEM: The current South Australian Link Emissions Model (CLEM) is used for all non-petrol passenger vehicles giving link emissions estimates, $L E_{i}^{C}$, as

$$
L E_{i}^{C}=s(t) \lambda \sum_{j} N_{j} e_{i j}(\bar{v})
$$

for pollutant $i$ from vehicles of type $j$, measured in g/s. In (1), $\lambda$ is the length of the road link, $N_{j}$ is the number of vehicles of type $j$ travelling on the link, $e_{i j}(\bar{v})$ is the emission factor describing the rate of emission of pollutant $i$ from vehicles of type $j$, and $s(t)$ is a proportion representing hourly variation in traffic volume on the link. The emission factors $e_{i j}(\bar{v})$ were developed for overseas conditions and are calibrated for use in South Australia (Stephenson and Giandomenico, 2010). CLEM estimates link emissions based on a single average vehicle speed of $\bar{v}=55.98 \mathrm{~km} / \mathrm{hr}$ thereby assuming all traffic flows at a constant rate; and

2. PLEM: We developed the petrol passenger vehicle Link Emissions Model (PLEM) using the NISE2 emissions data and the petrol passenger vehicle emission factors, $e_{i j}$, derived in Section 3.1. PLEM computes link emissions, $L E_{i}^{P}$, as

$$
L E_{i}^{P}=s(t) \lambda \sum_{j} N_{j} \sum_{v} \frac{e_{i j}(v)}{v} f(v)
$$

given in $\mathrm{g} / \mathrm{s}$. The emission factors $e_{i j}$ describe the rate of emission of pollutant $i$ from petrol passenger vehicles of type $j, v$ is the vehicle speed $(\mathrm{km} / \mathrm{hr})$ and $f(v)$ represents the vehicle speed distributions derived from the NISE2 CUEDC for residential, arterial and congested roads as well as freeways (Schultz et al., 2011). The main difference between the models in (1) and (2) is that PLEM accommodates a range of vehicle speeds and computes estimates based on Australian data and emissions conditions. 


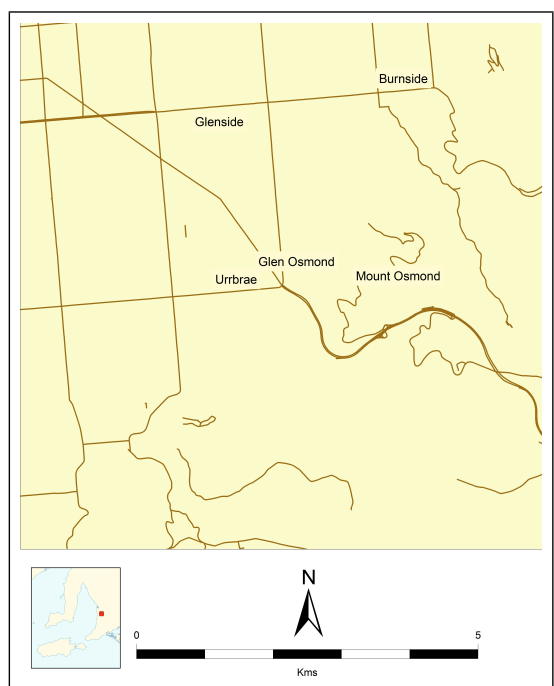

(a)

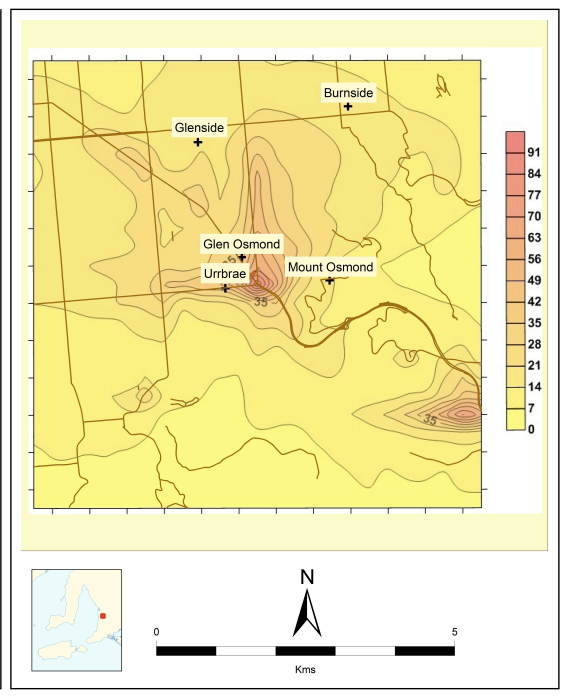

(b)

Figure 2. (a) Case study area in Adelaide. (b) Maximum estimated winter CO concentrations in $\mu \mathrm{g} / \mathrm{m}^{3}$.

\section{CASE STUDY: RESIDENTIAL AREA SOUTH OF ADELAIDE}

The case study area south of the Adelaide CBD (Figure 2(a)) combines residential zoning with heavily trafficked main roads, including a freeway. The typical vehicle fleet comprises passenger vehicles, buses and other high-polluting vehicles. As such, the case study area is a potential high TRAP exposure zone.

We arbitrarily selected a fortnight period from each season in 2009, which the South Australian Environment Protection Authority considers to be a typical meteorological year. The enhanced Link Emissions Model estimated TRAP emissions for the common exhaust pollutants CO, THC and NOx over the road links. Atmospheric dispersion of the pollutant estimates was analysed using TAPM and the output was combined with GIS data to generate pollutant exposure maps for each fortnight under consideration. The winter exposure map for the maximum estimated $\mathrm{CO}$ concentrations overlaying the case study road network is provided in Figure 2(b).

The greatest concentrations of $\mathrm{CO}$ occur over the intersection of the three main roads with the freeway, situated at the centre of Figure 2(b), and are also substantial near the freeway exit/entry ramp towards the bottom-right corner of the exposure map. Pollution concentrations decline with increasing distance from the main roads. The dispersion appears to be skewed to the west/north-west of the exposure map, indicating the wind was commonly easterly/south-easterly during the winter fortnight. This is a typical wind direction for the case study region as the low-lying area of the case study is situated immediately west of the Adelaide Hills.

Due to space constraints we have ommitted the exposure maps for NOx, THC and the other seasons, however all three pollutants share a common dispersion trend, with atmospheric concentrations being the most notable difference between each exposure map. There are two possible justifications for the shared dispersion trend. First, the only difference in the enhanced Link Emissions Model is the rate at which a particular pollutant is emitted from vehicular exhaust. Second, the research presented here is primarily focused on the meteorological effects on pollutant dispersion and as such, we are not considering dry or wet deposition of the pollutants or chemical reactions in the atmosphere. The pattern of pollutant dispersion for the three pollutants (Figure 2(b)) persisted across each season of the case study however there was variation in the estimated atmospheric pollution concentrations between seasons. Table 1 summarises the overall maximum estimated pollutant concentrations and the maximum of the average pollutant concentration estimates by season, from which it can be seen that winter has the highest pollutant concentrations. These concentrations are comfortably below the World Health Organization guideline thresholds. There is still enough pollutant exposure however, particularly near the main intersection at the centre of the case study (Figure 2(b)) to suggest residents may be at risk of experiencing adverse health effects associated with pollution exposure.

As this study specifically considers the influence of meteorology on pollutant dispersion, the variability in overall and average maximum pollutant concentrations is likely the result of wind speed. Figure 3 (a) con- 
L. Schultz et al, Modelling Motor Vehicle Emissions and Population Exposure in South Australia

Table 1. Seasonal maximum average and total maximum estimated pollutant concentrations in $\mu \mathrm{g} / \mathrm{m}^{3}$.

\begin{tabular}{lcccccc}
\hline \hline & \multicolumn{2}{c}{ CO } & \multicolumn{2}{c}{ NOx } & \multicolumn{2}{c}{ THC } \\
Season & Max avg & Overall max & Max avg & Overall max & Max avg & Overall max \\
\hline \hline Winter & 16 & 98 & 1 & 8 & 0.6 & 4.6 \\
Spring & 8 & 80 & 0.51 & 5.6 & 0.3 & 3.6 \\
Summer & 6 & 100 & 0.44 & 8 & 0.26 & 4.4 \\
Autumn & 10 & 120 & 0.7 & 9 & 0.4 & 5.5 \\
\hline \hline
\end{tabular}

tains a time series plot of wind speed for the winter case study and the corresponding CO concentrations (in $\mu \mathrm{g} / \mathrm{m}^{3}$ ). Periods of stagnant wind conditions, such as approximately the evening of Day 12, permit plumes of pollution to collect and linger in the atmosphere. Periods of high wind velocity, such as midday on Day 6 , rapidly displace pollution, resulting in accelerated atmospheric dispersion. Moreover, the higher pollutant

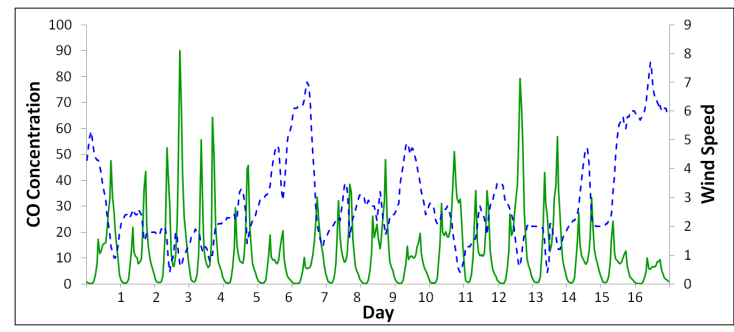

(a)

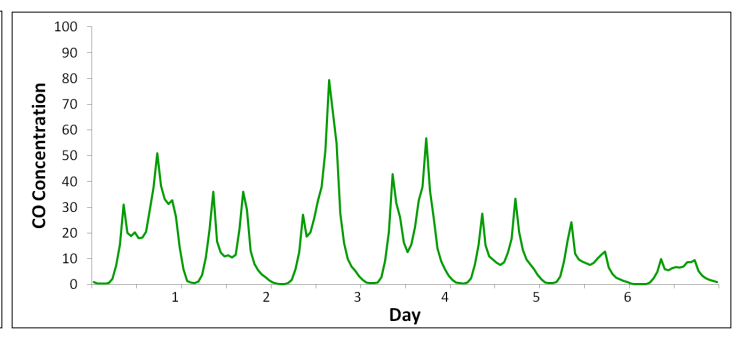

(b)

Figure 3. (a) Wind speed ( $\mathrm{m} / \mathrm{s},--)$ and the corresponding CO concentration $\left(\mu \mathrm{g} / \mathrm{m}^{3},-\right)$ for the winter case study; (b) Hourly variation in CO concentration over a one week period in the winter case study.

concentrations in winter are likely to be the result of typical cold weather conditions such as calmer wind and a lower mixing layer in the atmosphere which reduces pollutant dispersion potential. Conversely, summer has the lowest average maximum pollutant concentrations (Table 1). This is likely attributable to typically windier conditions and a higher mixing layer in warmer weather.

Figure 3(b) depicts a CO concentration time series for the winter case study, commencing on Day 1 (Thursday, June 11th, 2009) and finishing on Day 7 (Thursday, June 17th, 2009). Hourly CO concentrations appear to fluctuate across the week and each day of the week has two peaks in CO concentrations which coincide with peak traffic hours. The greatest estimated CO concentrations occur during Days 3 and 4 which are a Saturday and Sunday, respectively. These peaks are likely attributable to the increased weekend traffic in the case study area, with people taking day or weekend trips out of the city. For example, at the weekend Adelaide residents often visit the Adelaide Hills, located to the east/south-east of the case study area. Figure 3(b) also shows the lowest concentrations of $\mathrm{CO}$ occur during the early hours of each day, between approximately midnight and 6:00 am. This time corresponds to the lowest traffic volumes in a typical day.

\section{CONCLUSIONS \& FUTURE WORK}

We have enhanced the current South Australian Link Emissions Model through the derivation of Australian petrol passenger vehicle emission factors and the corresponding Link Emissions Model, PLEM. The use of PLEM in conjunction with CLEM extended the current South Australian emissions modelling procedure to consider a range of vehicle speeds based on Australian data. A case study investigating the current air quality status and population exposure levels in residential areas of Adelaide, South Australia, indicated meteorology has a strong effect on the pattern of pollution dispersion as well as seasonal, weekly and daily variability. Adelaide residents are exposed to a notable volume of TRAP concentrations, thus mitigation strategies for these emissions and exposure should be designed and implemented for South Australia. 
Future research will see the derivation of new emission factors and corresponding Link Emissions Models, for vehicle types other than petrol passenger vehicles, as the data becomes available as well as the use of chemical reactions in the atmosphere and dry/wet pollutant deposition in the dispersion analysis. Moreover, we will broaden the spectrum of pollutants considered in the case study analysis.

\section{ACKNOWLEDGEMENT}

This research would not have been possible without the continual support of the South Australian Environment Protection Authority to whom we would like to extend our sincere thanks and appreciation.

\section{REFERENCES}

Brauer, M., G. Hoek, P. van Vliet, K. Meliefste, P. Fischer, A. Wijga, L. Koopman, H. Neijens, J. Gerritsen, M. Kerkhof, J. Heinrich, T. Bellander, and B. Brunekreef (2002). Air pollution from traffic and the development of respiratory infections and asthmatic and allergic symptoms in children. American Journal of Respiratory and Critical Care Medicine 166, pp. 1092-1098.

Department of the Environment, Water, Heritage and the Arts (2009). Second National In-Service Emissions Study (NISE2), light duty petrol vehicle emissions testing. Final report, Australian Government.

Fenger, J. (1999). Urban air quality. Atmospheric Environment 33, pp. 4877-4900.

Gehring, U., A. Wijga, P. Fischer, J. de Jongste, M. Kerkhof, G. Koppelman, H. Smit, and B. Brunekreef (2011). Traffic-related air pollution, preterm birth and term birth weight in the PIAMA birth cohort study. Environmental Research 111, pp. 125-135.

HEI Panel on the Health Effects of Traffic-Related Air Pollution (2010). Traffic-related air pollution: A critical review of the literature on emissions, exposure, and health effects. HEI Special Report 17, Health Effects Institute, Boston, MA.

Hurley, P. (2008). TAPM V4. Part 1: Technical description. Technical Report 25, CSIRO Marine and Atmoshperic Research.

Loader, C. (1999). Local Regression and Likelihood. New York, USA: Springer.

Miller, K., D. Siscovick, L. Sheppard, K. Shepherd, J. Sullivan, G. Anderson, and J. Kaufman (2007). Longterm exposure to air pollution and incidence of cardiovascular events in women. The New England Journal of Medicine 356(5), pp. 447-458.

Oosterlee, A., M. Drijver, E. Lebret, and B. Brunekreef (1996). Chronic respiratory symptoms in children and adults living along streets with high traffic density. Occupational and Environmental Medicine 53, pp. 241-247.

Power, M., M. Weisskopf, S. Alexeef, B. Coull, A. Sipro, and J. Schwartz (2011). Traffic-related air pollution and cognitive function in a cohort of older men. Environmental Health Perspectives 119(5), pp. 682-687.

Raaschou-Nielsen, O., M. Sørensen, M. Ketzel, O. Hertel, S. Loft, A. Tjønneland, K. Overvad, and Z. Andersen (2013). Long-term exposure to traffic-related air pollution and diabetes-associated mortality: A cohort study. Diabetologia 56, pp. 36-46.

Schultz, L., B. Chiera, P. Shah, and E. Giandomenico (2011). Modelling motor vehicle emissions in Australia: The effects of vehicle speed on air quality emissions. Honours thesis, University of South Australia.

Scoggins, A., T. Kjellstrom, G. Fisher, J. Connor, and N. Gimson (2004). Spatial analysis of air pollution exposure and mortality. Science of the Total Environment 321, pp. 71-85.

Stephenson, P. and E. Giandomenico (2010). A summary of the EPA University of South Australia Motor Vehicle Emissions Inventory model. Technical report, South Australian office of the Environment Protection Authority.

Van Mierlo, J., G. Maggetto, E. van de Burgwal, and R. Gense (2004). Driving style and traffic measures influence on vehicle emissions and fuel consumption. Proceedings of the Institution of Mechanical Engineers 218(1), pp. 43-50. 